\title{
The transcription factor MITF is a critical regulator of GPNMB expression in dendritic cells
}

\author{
Michael Gutknecht, Julian Geiger, Simone Joas, Daniela Dörfel, Helmut R Salih, Martin R Müller,
} Frank Grünebach ${ }^{*}$ and Susanne M Rittig

\begin{abstract}
Background: Dendritic cells (DC) are the most potent antigen-presenting cells (APC) with the unique ability to activate naive $T$ cells and to initiate and maintain primary immune responses. Immunosuppressive and anti-inflammatory stimuli on DC such as the cytokine IL-10 suppress the activity of the transcription factor NF-KB what results in downregulation of costimulatory molecules, MHC and cytokine production. Glycoprotein NMB (GPNMB) is a transmembrane protein, which acts as a coinhibitory molecule strongly inhibiting T cell responses if present on APC. Interestingly, its expression on human monocyte-derived dendritic cells (moDC) is dramatically upregulated upon treatment with IL-10 but also by the BCR-ABL tyrosine kinase inhibitors (TKI) imatinib, nilotinib or dasatinib used for the treatment of chronic myeloid leukemia (CML). However, the molecular mechanisms responsible for GPNMB overexpression are yet unknown.
\end{abstract}

Results: The immunosuppressive cytokine IL-10 and the BCR-ABL TKI imatinib or nilotinib, that were examined here, concordantly inhibit the PI3K/Akt signaling pathway, thereby activating the downstream serine/threonine protein kinase GSK3ß, and subsequently the microphthalmia-associated transcription factor (MITF) that is phosphorylated and translocated into the nucleus. Treatment of moDC with a small molecule inhibitor of MITF activity reduced the expression of GPNMB at the level of mRNA and protein, indicating that GPNMB expression is in fact facilitated by MITF activation. In line with these findings, PI3K/Akt inhibition was found to result in GPNMB overexpression accompanied by reduced stimulatory capacity of moDC in mixed lymphocyte reactions (MLR) with allogeneic $T$ cells that could be restored by addition of the GPNMB T cell ligand syndecan-4 (SD-4).

Conclusions: In summary, imatinib, nilotinib or IL-10 congruently inhibit the PI3K/Akt signaling pathway thereby activating MITF in moDC, resulting in a tolerogenic phenotype. These findings extend current knowledge on the molecular mechanisms balancing activating and inhibitory signals in human DC and may facilitate the targeted manipulation of T cell responses in the context of DC-based immunotherapeutic interventions.

Keywords: Dendritic cells, Coinhibitory receptor, Glycoprotein NMB, Tyrosine kinase inhibitors, PI3K/Akt signaling pathway, Microphthalmia-associated transcription factor

\section{Background}

Pathways involved in negative $\mathrm{T}$ cell regulation are of great interest, as on the one hand they can fatally attenuate $\mathrm{T}$ cell responses against cancer cells and on the other hand do offer an opportunity to develop tolerance-inducing strategies in Graft-versus-host disease (GvHD) and autoimmune diseases $[1,2]$.

DC are the most powerful APC and play a key role in balancing $\mathrm{T}$ cell responses, depending on their expression

\footnotetext{
* Correspondence: Frank.Gruenebach@med.uni-tuebingen.de Department of Internal Medicine II, Oncology, Hematology, Immunology, Rheumatology and Pulmology, University of Tübingen, Otfried-Müller-Str. 10, 72076 Tübingen, Germany
}

of costimulatory and/or coinhibitory molecules [3]. After stimulation by TLR ligands, TNF, IFN- $\gamma$ or T cell signals, DC undergo a complex maturation process, express costimulatory molecules and migrate into lymph nodes where they prime naive $T$ cells. In contrast, in the absence of activating signals and/or in the presence of immunosuppressive and anti-inflammatory factors like IL-10, TGF- $\beta$, prostaglandin D2 (PGD2) or corticosteroids, DC achieve a tolerogenic phenotype mediated by the expression of molecules that suppress $\mathrm{T}$ cell activation and induce $\mathrm{T}$ cell anergy $[3,4]$. Due to their unique ability to induce specific T cell responses DC are employed in immunotherapeutic 
strategies against cancer aiming at the induction of long term clinical responses [5-7].

At the same time, targeted therapies with TKI have significantly improved treatment of cancer with imatinib being the first to be established in the treatment of chronic myeloid leukemia (CML). It efficiently blocks the pathologically activated c-ABL tyrosine kinase activity of the BCR-ABL fusion oncogene [8-10]. Nilotinib and dasatinib, secondgeneration TKI initially developed for the treatment of patients who are resistant or intolerant to imatinib, are now used as first-line therapy [11-13]. Besides c-ABL, these TKI significantly inhibit c-Kit and PDGFR tyrosine kinase activity and imatinib therefore is being used against other malignancies including gastrointestinal stromal tumors. However, little is known about their effects on immune cells.

Recently, the type I transmembrane receptor GPNMB (Glycoprotein NMB, DC-associated transmembrane protein (DC-HIL), osteoactivin), expressed on APC, was shown to strongly inhibit responses of $\mathrm{CD}_{4}^{+}$and $\mathrm{CD}^{+} \mathrm{T}$ cells by binding to its ligand syndecan-4 (SD-4) [14-18]. We previously demonstrated that primary human moDC moderately express GPNMB and dramatically upregulate its expression if generated in the presence of the cytokine IL-10, a main suppressor of cellular immunity, but notably also when exposed to imatinib, nilotinib or dasatinib $[19,20]$.

Here we aimed to elucidate the molecular switch of cellular signaling upon inhibition of moDC. Our in vitro study revealed concordant inhibition of PI3K/Akt signaling by IL-10 or the BCR-ABL TKI imatinib and nilotinib that resulted in dephosphorylation and activation of glycogen synthase kinase-3-ß (GSK3ß) and subsequent phosphorylation and translocation of the transcription factor MITF [21]. Moreover, treatment of moDC with the small molecule inhibitor of the MITF molecular pathway ML329 [22] reduced the expression of GPNMB at the level of mRNA and protein, indicating that GPNMB expression is in fact facilitated by MITF activation.

The basic helix-loop-helix leucine zipper transcription factor MITF, which was initially described as a key regulator for melanocyte differentiation, comprises at least eight isoforms differentially expressed within various cell types $[21,23]$. However, its expression pattern and functional role in hematopoietic and blood cells was so far unknown.

Finally, PI3K/Akt inhibition was found to result in GPNMB overexpression accompanied by reduced stimulatory capacity of moDC in mixed lymphocyte reactions (MLR) with allogeneic $\mathrm{T}$ cells that could be restored by addition of the T cell ligand SD-4, demonstrating the functional relevance of the elucidated signaling mechanism.

Taken together, our data indicate that the therapeutically used BCR-ABL TKI imatinib and nilotinib exert immunosuppressive effects in primary moDC by interfering with pathways involved in IL-10 receptor signaling and activation of MITF. These findings extend the current knowledge about the molecular mechanisms balancing between activating and inhibitory signals in DC and, thus, could help to avoid impaired immune responses due to TKI treatment. In addition, manipulation of the relevant signaling cascades and/or GPNMB expression or function may constitute a promising strategy in combinatory approaches using BCR-ABL TKI and DC-based immunotherapy and may also allow for manipulation of $\mathrm{T}$ cell responses in GvHD.

\section{Results}

\section{PI3K/Akt-Inhibition upregulates GPNMB expression in moDC}

Besides BCR-ABL, imatinib, nilotinib and dasatinib inhibit a variety of other kinases including c-Kit [24]. The main downstream signaling cascades are the Ras/Erkand the PI3K/Akt pathway. Evidence that IL-10 receptor signaling could be affected by these clinically used TKI is deduced from the observation in mouse DC that IL10 blocks Akt phosphorylation, and inhibitors of PI3K effectively suppress the activation of Akt and subsequent

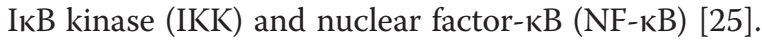

In our first experiments, the relevance of these pathways in (up-) regulation of immune repressive GPNMB in human DC was examined. Therefore, we generated immature moDC in vitro from $\mathrm{CD}_{1} 4^{+}$monocytes of healthy donors, incubated with the PI3K inhibitor LY294002, Akt inhibitor MK2206, Erk inhibitor FR180204 or imatinib or nilotinib as a control. GPNMB expression was determined by qRT-PCR and FACS analysis at day 7 of cell culture.

Consistent with our previous findings, incubation with BCR-ABL TKI imatinib or nilotinib from the first day of culturing resulted in a marked increase of GPNMB steady-state mRNA concentrations (Figure 1A) and cell surface protein (Figure 1B) on $\mathrm{CD}^{209^{+}}\left(\mathrm{DC}^{\mathrm{SIGN}}{ }^{+}\right)$ moDC. Interestingly, treatment of cells with 125-1000 nM Akt inhibitor or $500-1000 \mathrm{nM}$ of PI3K inhibitor also led to upregulation of GPNMB expression (Figure 1A, B and Additional file 1: Figure S1). In contrast, inhibition of the Erk-pathway by FR180204, c-Raf inhibitor 553008 or MEK1/2 inhibitors U0126 and PD0325901 did not have any significant effect on GPNMB expression (Figure 1A and $B$ or data not shown). In response to triggering TLR4 signaling by lipopolysaccharide (LPS), Akt is phosphorylated rapidly through PI3K [26]. In accordance with this mechanism and our previous findings [20], stimulation of moDC with LPS resulted in downregulation of GPNMB expression and compensated nilotinib-induced upregulation of GPNMB cell surface protein (Figure 1C).

Immunophenotyping using flow cytometry (FACS) revealed that moDC treated with nilotinib or Akt inhibitor consistently retained a more $\mathrm{CD} 14^{+}$phenotype and exhibited reduced expression of the DC marker CD1a as 
compared to untreated cells (Figure 1D) indicating inhibition of full cellular differentiation. Other typical surface markers necessary for $\mathrm{T}$ cell activation, such as CD80, CD86 or the DC-specific adhesion receptor DCSIGN (CD209), were not consistently affected by the different treatments (data not shown). Administration of $300 \mathrm{nM}$ Akt inhibitor slightly increased the percentage of dead cells by an average of $3.5 \%$ in comparison with untreated cells (data not shown).

Combined, these experiments demonstrate the functional involvement of the PI3K/Akt pathway in the regulation of the expression of the inhibitory molecule GPNMB in moDC.

\section{The BCR-ABL TKI imatinib and nilotinib or IL-10 inhibit phosphorylation of Akt in moDC}

Recently, we showed that imatinib affects phenotype, cytokine secretion, and $\mathrm{T}$ cell stimulatory capacity of moDC due to the inhibition of NF-kB and Akt signaling pathways [27]. To analyze the relevance of PI3K/Akt signaling for the regulation of GPNMB expression, we examined the protein levels of Akt as well as its phosphorylation status in moDC by western blotting. To this end, moDC were generated from different donors, in the presence of imatinib, nilotinib, Akt inhibitor MK2206 or the immunosuppressive cytokine IL-10 as a positive control. GM-CSF and IL-4 activate the PI3K/Akt signaling pathway in monocytes which is critical for differentiation and generation of immature moDC [28]. To keep this pathway active, $\mathrm{CD}_{209^{+}}$purified cells were further incubated for 20 and/ or $40 \mathrm{~min}$ under the same cell culture conditions as on day 0 to restore the initial conditions prior to lysis.

Incubation with imatinib (Figure 2A) or nilotinib (Figure 2B) resulted in decreased amounts of phosphorylated Akt as compared to the respective untreated controls within the indicated timeframe, while the levels of total Akt remained unchanged. As shown in Figure 2C MK2206 (Akt-inh., $300 \mathrm{nM}$ ) very effectively inhibited Akt phosphorylation after $40 \mathrm{~min}$ in $\mathrm{moDC}$. These findings were approved for IL-10 and TKI with moDC generated from blood monocytes of an additional donor (Figure 2D and Additional file 2: Figure S2). Furthermore, western blot analysis confirmed the FACS data (Figure 1B, C) and revealed a pronounced increase of GPNMB protein levels upon treatment with imatinib or Akt-inhibitor MK2206 (Figure 2E) as well as nilotinib or IL-10 (Figure 2F). As shown in Figure 2G, stimulation of moDC with LPS efficiently compensated imatinib-induced upregulation of GPNMB.

In line with our previous findings these experiments indicate that BCR-ABL TKI and the immunosuppressive cytokine IL-10 concordantly inhibit the phosphorylation of Akt in immature moDC and suggest that Akt dephosphorylation is critically involved in the upregulation of the inhibitory receptor GPNMB.

\section{Imatinib, nilotinib, Akt inhibitor or IL-10 prevent phos- phorylation of GSK3B in moDC}

A central issue of our study was to elucidate the molecular switch that facilitates transcriptional activation upon BCRABL TKI- or IL-10-mediated inhibition of cellular signaling in moDC. Accordingly, we focused our further analyses on proteins that promote gene transcription upon inhibition by Akt. Such a downstream molecule of the PI3K/Akt pathway is the serine/threonine protein kinase GSK3 $\beta$ that phosphorylates a broad range of substrates, including several transcription factors. When the PI3K/ Akt pathway is active, GSK3 $\beta$ is inhibited as a result of Akt phosphorylation. Conversely, inhibition of PI3K/Akt signaling results in dephosphorylation and activation of GSK3 $\beta[29,30]$. Therefore, we next investigated the possibility that the inhibition of Akt by TKI or IL-10 results in dephosphorylation and thereby activation of GSK3 $\beta$ in moDC. For the respective western blot analysis a monoclonal antibody that detects both isoforms of $\operatorname{GSK} 3(\alpha, \beta)$ was used. Consistent with the hypothesized mechanism, we detected substantially lower amounts of phosphorylated GSK3 $\beta$ (upper panels, lower band, $46 \mathrm{kDa}$ ) in the samples treated with imatinib (Figure 3A), nilotinib (Figure 3B) or Akt inhibitor MK2206 (Figure 3C) as compared to untreated controls. These experiments were repeated for IL-10 and TKI with moDC generated from blood monocytes of an additional donor (Figure 3D and Additional file 3: Figure S3). The phosphorylation status of GSK3 $\alpha$ (Figure 3A-D: upper bands, $51 \mathrm{kDa}$ ) as well as the level of unphosphorylated GSK3 $\beta$ (Figure 3A-D: lower panels) was not affected by the treatment of moDC.

These experiments indicate that inhibition of the Akt signaling pathway by BCR-ABL TKI, MK2206 or IL-10 in developing human moDC results in reduced phosphorylation and subsequent activation of GSK3 $\beta$ and suggest a mechanism, by which inhibition of cell signaling could induce transcriptional activation of immune inhibitory molecules such as GPNMB.

\section{The transcription factor MITF is expressed in progenitor cells, leucocytes and primary moDC}

Next we aimed to identify the responsible transcription factor downstream of the PI3K/Akt signaling cascade which is activated by GSK3 3 . Interestingly, GPNMB expression in melanoblasts and osteoclasts was shown to be dependent on MITF, and in human glioblastoma cells this transcription factor is activated by PI3K/Akt and GSK3 $\beta$ signaling [31,32]. However, its expression pattern and functional role in hematopoietic and blood cells is so far unknown. Therefore, we first examined the expression of MITF mRNA in hematopoietic and blood cells by qRTPCR. Significant expression was observed only in CD14 ${ }^{+}$ monocytes and $\mathrm{CD}_{4} 4^{+}$progenitor cells. $\mathrm{CD} 4^{+}, \mathrm{CD} 8^{+}$and 

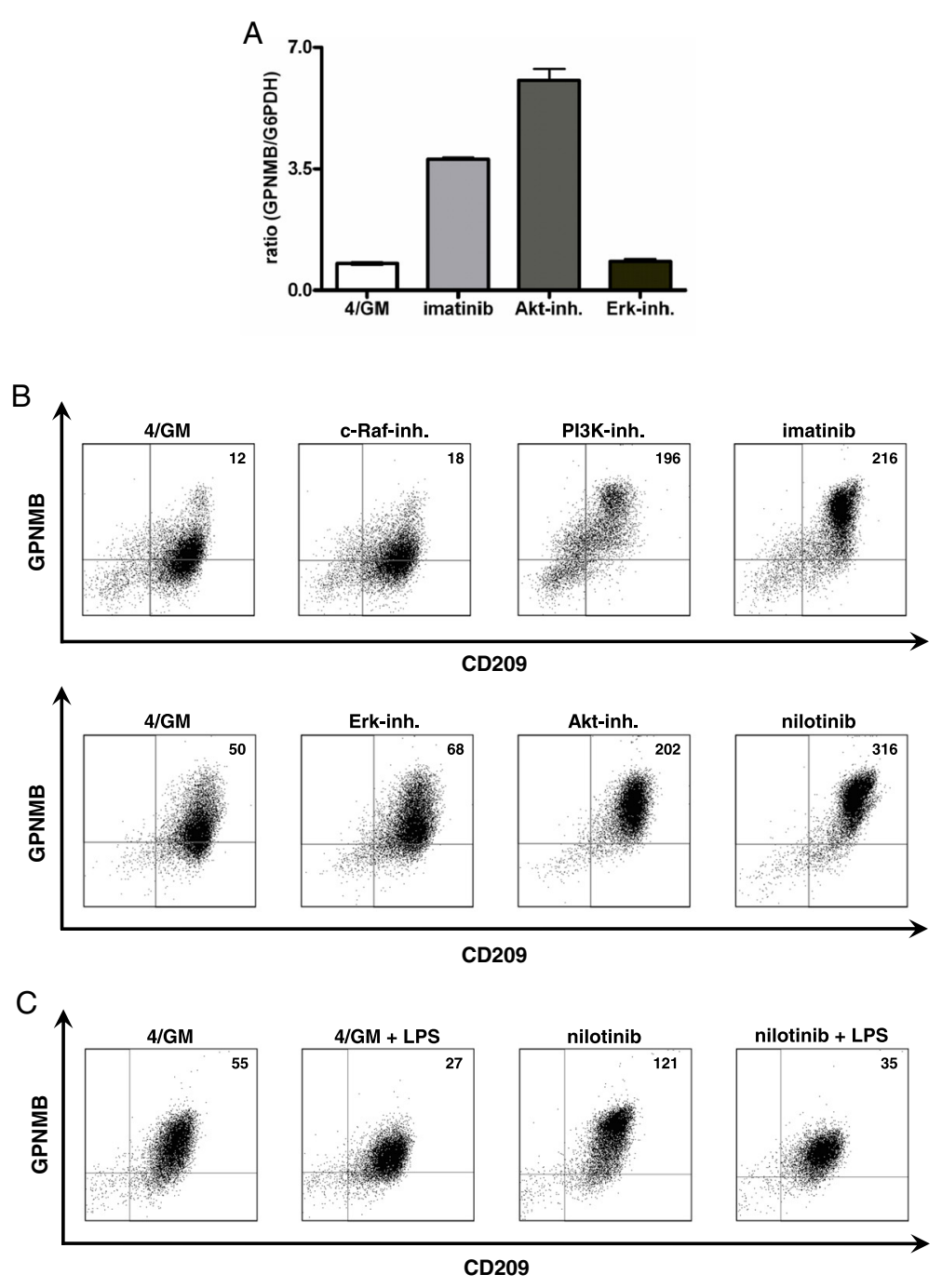

D

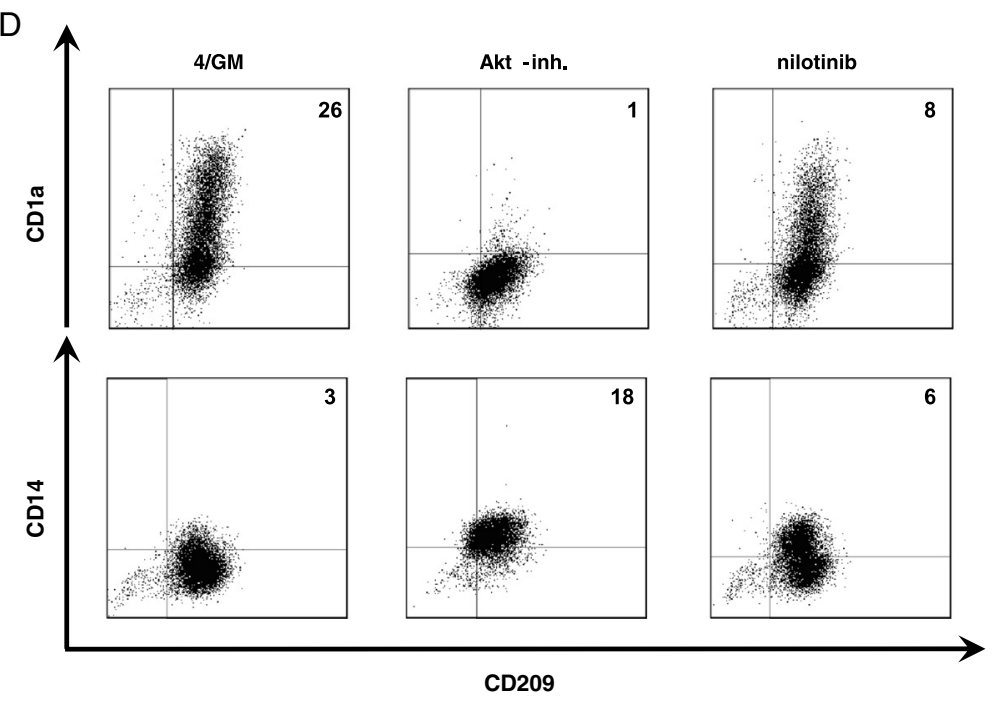

Figure 1 (See legend on next page.) 
(See figure on previous page.)

Figure 1 PI3K/Akt-inhibition upregulates GPNMB gene expression in human moDC. Immature moDC were generated in vitro with GM-CSF and IL-4 alone (4/GM) or with additional TKI (3 $\mu \mathrm{M}$ imatinib or $3 \mu \mathrm{M}$ nilotinib) or inhibitors of signal transduction (300 nM Akt inhibitor MK2206 (Akt-inh.), 300 nM Erk inhibitor FR180204 (Erk-inh.), 100 nM PI3K inhibitor LY294002 (PI3K-inh.), 20 nM c-Raf inhibitor 553003 (c-Raf-inh.)) and analyzed for GPNMB expression. Exemplary results from at least three independent experiments using different donors are presented. (A) qRT-PCR analysis: relative level of GPNMB mRNA. The mean $\left( \pm\right.$ SD) of duplicate measurements is shown. (B, C) GPNMB protein level of CD209 ${ }^{+}$moDC (of three different donors) was analyzed by flow cytometry. Where indicated, maturation of moDC was induced by LPS. Data were analyzed using FlowJo software and Difference in Median Fluorescence Intensity (DMFI) of CD209+ cells is shown in the upper right quadrants. (D) Phenotypic changes of immature moDC in the absence $(4 / G M)$ or presence of nilotinib or Akt inhibitor were analyzed by flow cytometry. Double stainings were performed with monoclonal antibodies recognizing CD209, CD1a or CD14. DMFI of CD209 cells is shown in the upper right quadrants.

$\mathrm{CD} 19^{+}$cells showed very low and $\mathrm{CD} 4^{+} \mathrm{CD} 25^{+}$regulatory T cells no expression of MITF (Figure 4A). In our next set of experiments, we analyzed MITF mRNA levels in immature purified $\mathrm{CD}_{209^{+}}$moDC. As shown in Figure 4B, transcripts were detected in untreated cells as well as in samples treated with TKI or IL-10. Notably, steady-state transcript levels increased upon treatment with imatinib, nilotinib or IL-10 and positively correlated with the GPNMB mRNA expression in these cells (Figure 4D and Additional file 4: Figure S4). Interestingly, among the leucocytes analyzed, GPNMB mRNA was detected only in $\mathrm{CD}_{1}{ }^{+}$monocytes used for in vitro generation of moDC
(Figure 4C). However, moDC displayed significantly higher levels of expression than $\mathrm{CD}_{1} 4^{+}$cells (Figure 4D).

Next we determined MITF protein expression in purified $\mathrm{CD} 209^{+}$moDC by western blotting. In line with the mRNA expression pattern, we observed MITF protein in all analyzed cell extracts. Multiple bands representing the various isoforms were detected, of which the most prominent migrated at approximately 52 and $56 \mathrm{kDa}$ (Figure 4E). Due to lack of available phosphospecific antibodies for MITF, the phosphorylated and therefore activated form could only be detected by mobility shift. Western blotting revealed an additional, slower migrating
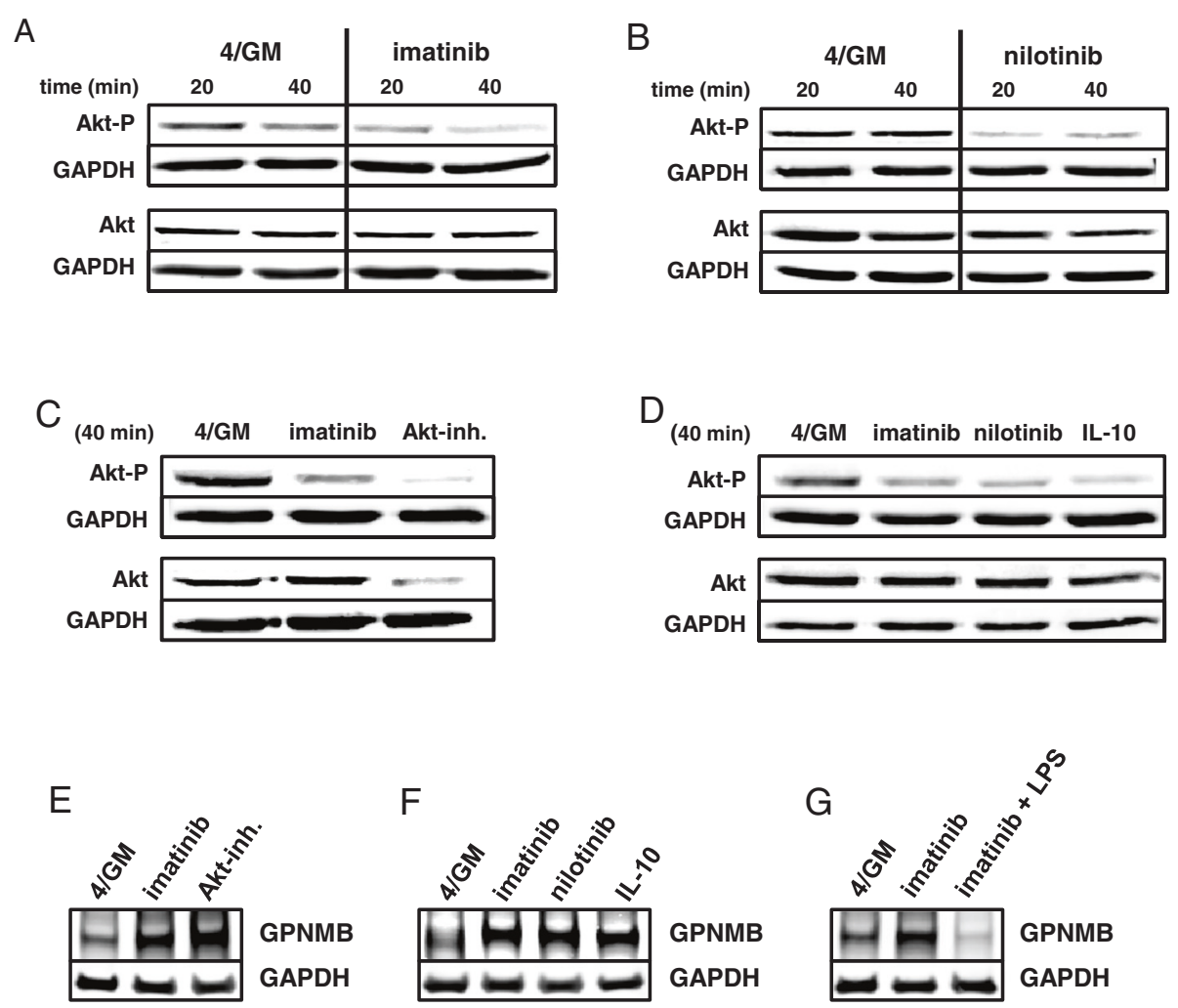

Figure 2 The BCR-ABL TKI imatinib and nilotinib or IL-10 inhibit phosphorylation of Akt in human moDC. Western blot analysis of total Akt levels and its phosphorylated form in purified immature CD209+ moDC (of four different donors). moDC were generated in vitro with GM-CSF and IL-4 alone (4/GM) or with additional (A) imatinib (3 $\mu \mathrm{M})$, (B) nilotinib $(3 \mu \mathrm{M})$, (C) Akt inhibitor MK2206 (Akt-inh., 300 nM) or (D) IL-10 $(10 \mathrm{ng} / \mathrm{mL}$ ). Indicated time refers to further treatment of cells prior to cell lysis (see Methods). (E-G) GPNMB protein levels in moDC were analyzed by western blotting. GAPDH served as loading control. Exemplary results from at least three independent experiments using different donors are presented. 

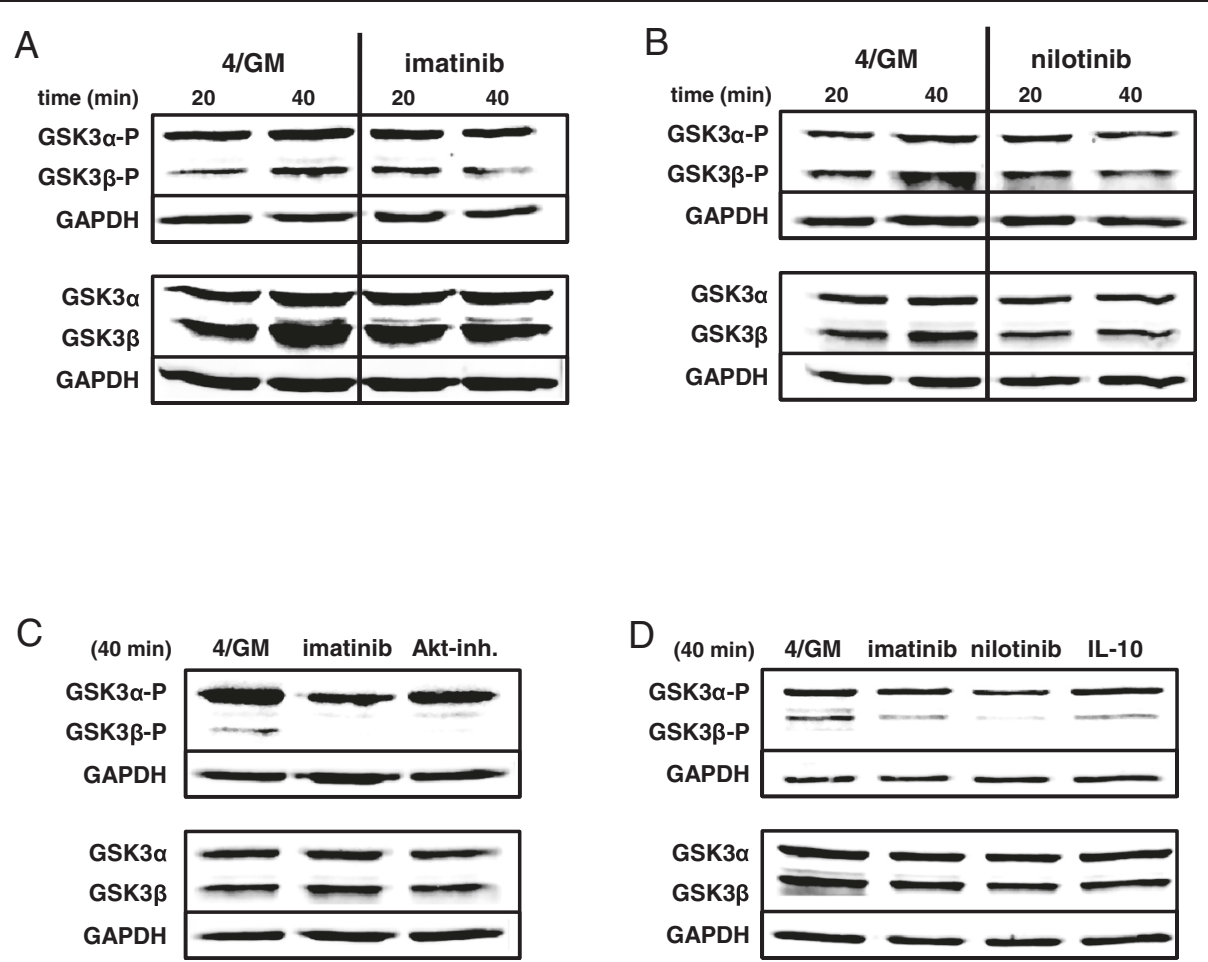

Figure 3 Imatinib, nilotinib, IL-10 or Akt inhibitor prevent phosphorylation of GSK3ß in human moDC. Western blot analysis of total GSK3B and GSK3a, as well as their phosphorylated forms in purified immature CD209+ moDC (of four different donors). moDC were generated

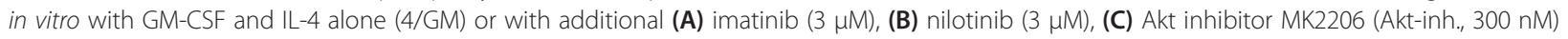
or (D) IL-10 (10 ng/mL). Indicated time refers to further treatment of cells prior to cell lysis (see Methods). GAPDH served as loading control. Exemplary results from at least three independent experiments using different donors are presented.

band that supposedly represents the phosphorylated protein of higher molecular weight at approximately $70 \mathrm{kDa}$ (Figure 4E).

Thus our data show that the transcription factor MITF is expressed in both $\mathrm{CD} 14^{+}$monocytes (the starting cells for the in vitro generation of moDC) and primary human immature moDC.

\section{MITF is phosphorylated in moDC after BCR-ABL TKI or IL- 10 treatment}

In a previous study GSK3 was found to phosphorylate serine 298 of MITF, thereby enhancing the binding to the tyrosinase promoter [33]. When analyzing MITF protein in whole cell lysates of moDC by western blotting, the additional slower migration band was clearly increased after $20 \mathrm{~min}$ in the samples incubated with nilotinib or IL-10 as compared to the untreated control (Figure 4E: lanes 2, 6, 7: upper band, approximately $70 \mathrm{kDa}$ ). To confirm that the shift in mobility was due to phosphorylation, cell lysates of moDC generated in the presence or absence of nilotinib were incubated with phosphatase. We found that such treatment reduced the intensity of the $70 \mathrm{kDa}$ band (Figure 4E, lane 4) when compared to untreated samples (Figure 4E, lane 2 and 6), thus confirming that the mobility shift described above was due to phosphorylation of
MITF. To further verify the specificity of the anti-MITF antibody, the HeLa cell line that reportedly exhibits a prominent MITF-band of $56 \mathrm{kDa}$ was included as a control (Figure 4E: lane 8).

Phosphorylated MITF translocates into the nucleus upon treatment of moDC with imatinib, nilotinib, IL-10 or Akt inhibitor

MITF contains a nuclear localization signal (NLS) and was shown to shuttle between cytoplasmic and nuclear compartments [34]. Therefore, we prepared cytoplasmic and nuclear extracts of the differently treated in vitro generated moDC and evaluated the localization of MITF. Western blot analyses of nuclear extracts revealed appearance of phosphorylated MITF after 20 to $40 \mathrm{~min}$ in cells generated in the presence of imatinib (Figure 5A: right panel), nilotinib (Figure 5B: right panel) or Akt inhibitor MK2206 (Figure 5C: right panel) indicating nuclear translocation in response to these stimuli. These findings were confirmed for IL-10 and TKI with moDC generated from blood monocytes of an additional donor (Figure 5D: right panel). In contrast, in the cytoplasmic fractions of the respective moDC populations, only unphosphorylated protein was detected (Figure 5A-D: left panels). 


\section{A}

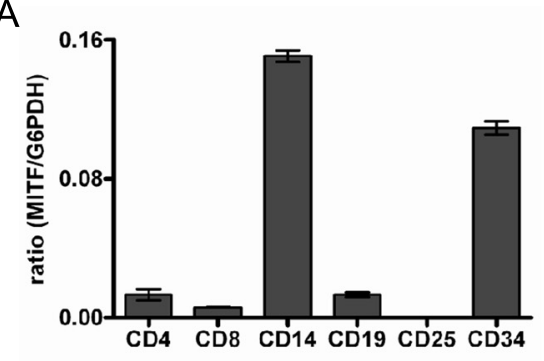

C

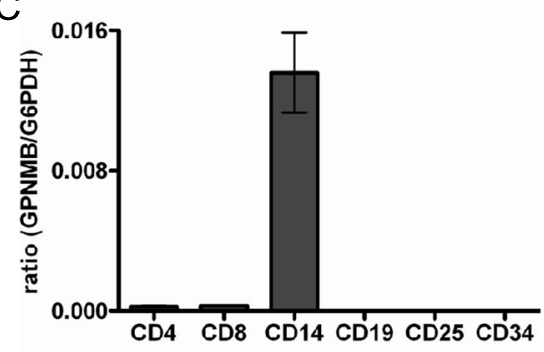

B
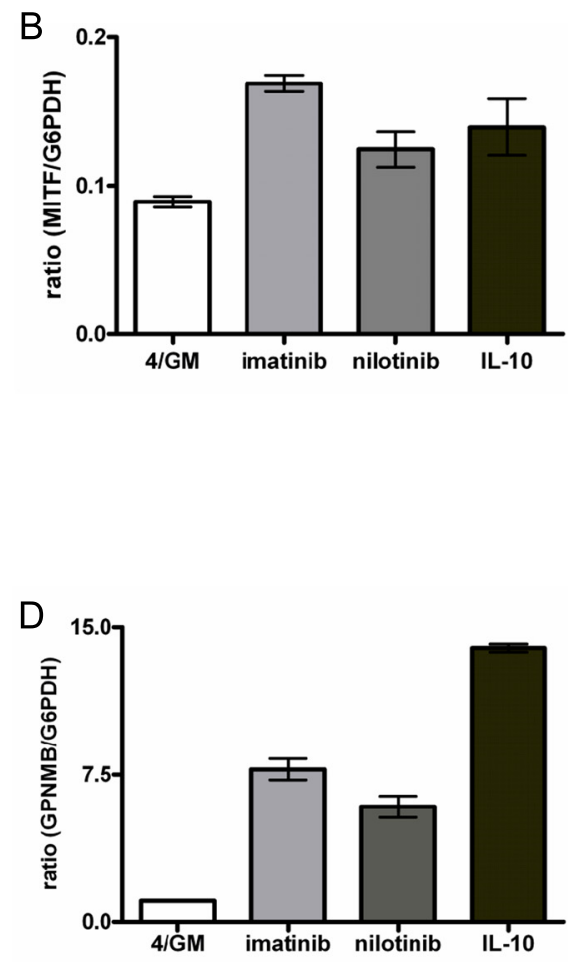

$\mathrm{E}$

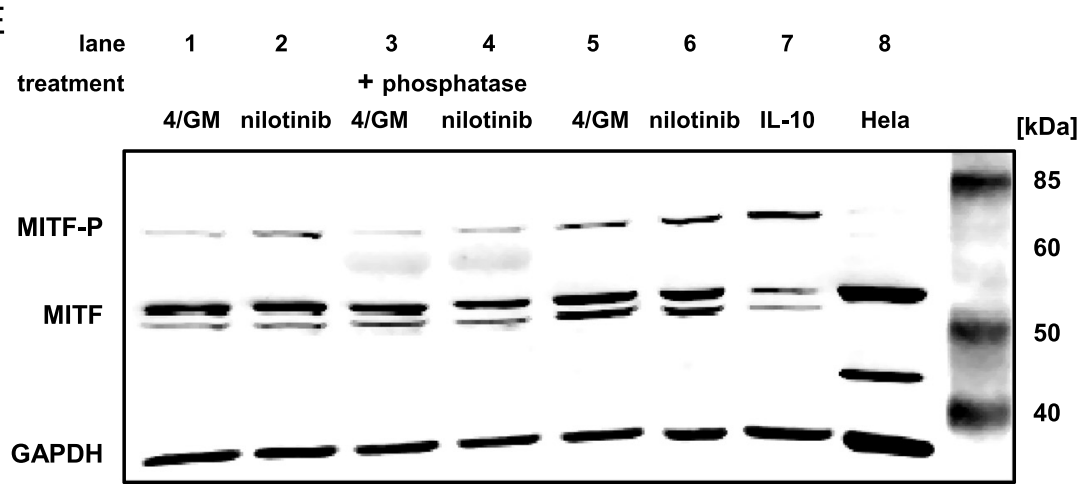

Figure 4 The transcription factor MITF is expressed in progenitor cells, leucocytes and primary moDC. Immature moDC were generated in vitro with GM-CSF and IL-4 alone (4/GM) or with additional TKI (3 $\mu \mathrm{M}$ imatinib or $3 \mu \mathrm{M}$ nilotinib) or IL-10 (10 ng/mL). For the analysis of CD34 ${ }^{+}$ progenitor and blood cells, cell-type specific total RNA was used. qRT-PCR analysis: (A, B) relative level of MITF and (C, D) GPNMB mRNA. The mean $( \pm$ SD) of duplicate measurements is shown. (E) MITF protein level and phosphorylation status was analyzed by western blotting in two different donors (lanes 1-4 and lanes 5-7, respectively). Phosphorylated MITF was detected by mobility shift (slower migrating band at $70 \mathrm{kDa}$ ). Western blotting revealed an additional, slower migrating band that supposedly represents the phosphorylated protein of higher molecular weight of approximately 70 kDa. "+ phosphatase": cell lysates were incubated with phosphatase. GAPDH served as loading control. Exemplary results from at least three independent experiments using different donors are presented.

Active signaling in the nucleus is terminated by dephosphorylation of transcription factors. In our experiments, the phosphorylated MITF band disappeared between 40 and $60 \mathrm{~min}$ as shown exemplary for nilotinib treated cells (Figure 5E: right panel).
Taken together, our results show for the first time that the transcription factor MITF is phosphorylated and translocated into the nucleus upon inhibition of the PI3K/Akt signaling cascade by clinically used BCR-ABL TKI or the immunosuppressive cytokine IL-10 in moDC. 

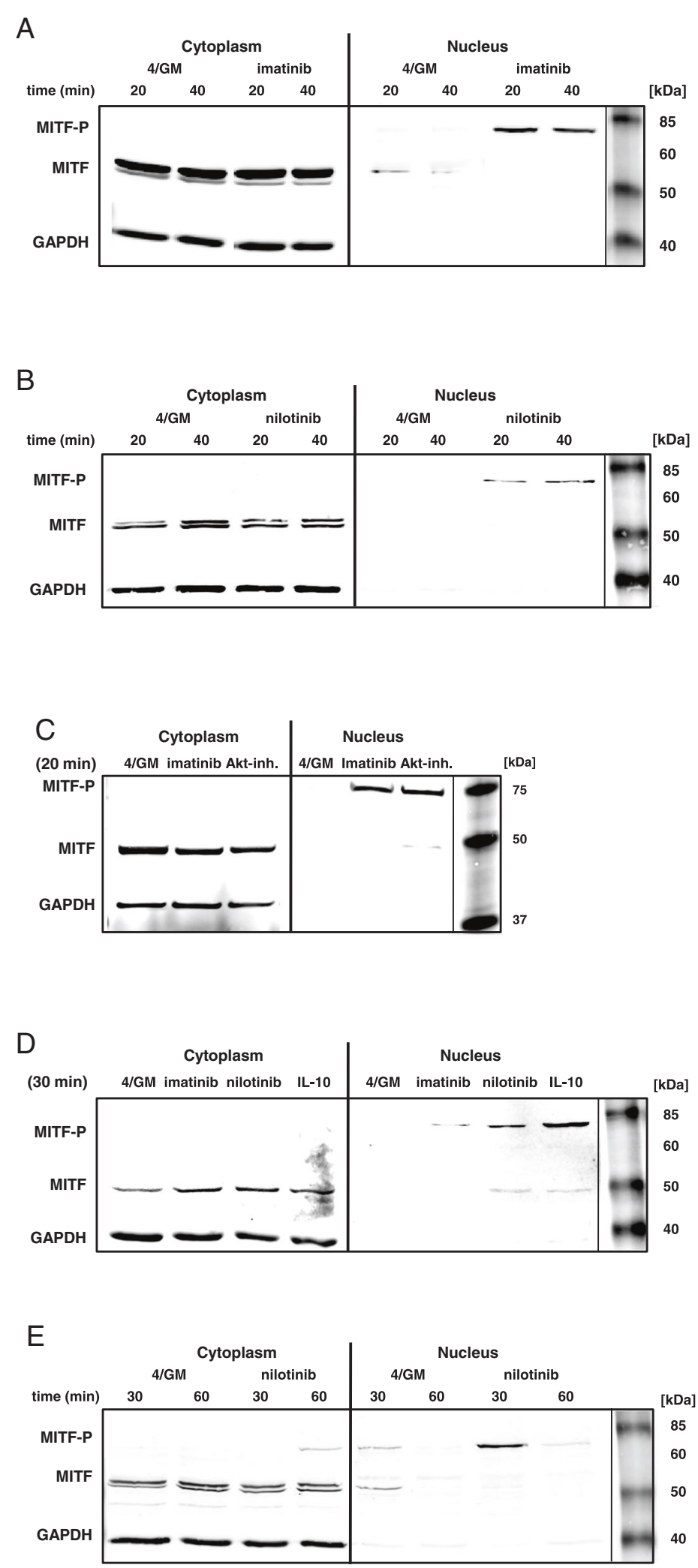

Figure 5 (See legend on next page.) 
(See figure on previous page.)

Figure 5 Upon treatment of moDC with imatinib, nilotinib, IL-10 or MK2206, MITF translocates into the nucleus. Western blot analysis of MITF level and phosphorylation status in the cytoplasmic or nuclear fraction of purified immature CD209 ${ }^{+}$moDC. moDC were generated in vitro with GM-CSF and IL-4 alone (4/GM) or with (A) imatinib (3 $\mu \mathrm{M})$, (B) nilotinib $(3 \mu \mathrm{M})$, (C) Akt inhibitor MK2206 (Akt-inh.; $300 \mathrm{nM})$ or (D) IL-10 (10 ng/mL). (E) Cells were treated with nilotinib $(3 \mu \mathrm{M})$. Indicated time refers to further treatment of cells prior to cell lysis (see Methods). GAPDH served as loading control. Exemplary results from at least three independent experiments using different donors are presented.

In the nucleus MITF is supposed to activate gene expression of inhibitory molecules such as GPNMB.

\section{MITF regulates GPNMB expression in moDC}

To directly verify the existence of a functional link between MITF and GPNMB expression in moDC, we used the small molecule inhibitor of MITF activity ML329 [22]. To that end, moDC were generated with or without IL-10 in the presence of ML329 or the solvent DMSO alone or CID-5951923 (KLF5 inhibitor) as controls. Importantly, treatment of cells with up to 2000 nM ML329 did neither alter their typical phenotype nor induce apoptosis as analyzed by immune phenotyping and Annexin-V/PI staining (data not shown). As shown in Figure 6A (and Additional file 5: Figure S5), incubation with increasing amounts of ML329 led to a gradual decline of the basal GPNMB mRNA levels to an average of $~ 30 \%$ with 2000 nM ML329 as compared to the DMSO or CID-5951923 control. Remarkably, even in the samples treated with IL-10, where GPNMB was strongly upregulated, mRNA levels were significantly reduced to an average of $13 \%$ with 2000 nM ML329 (Figure 6B and Additional file 5: Figure S5). Decrease of GPNMB at the protein level was confirmed by western blot analyses for moDC generated with
A

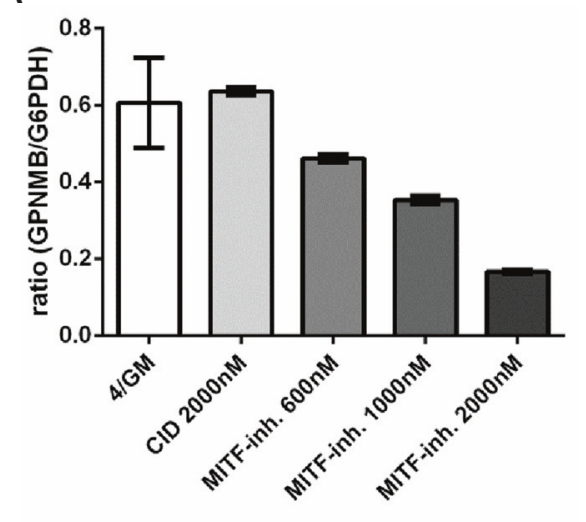

B

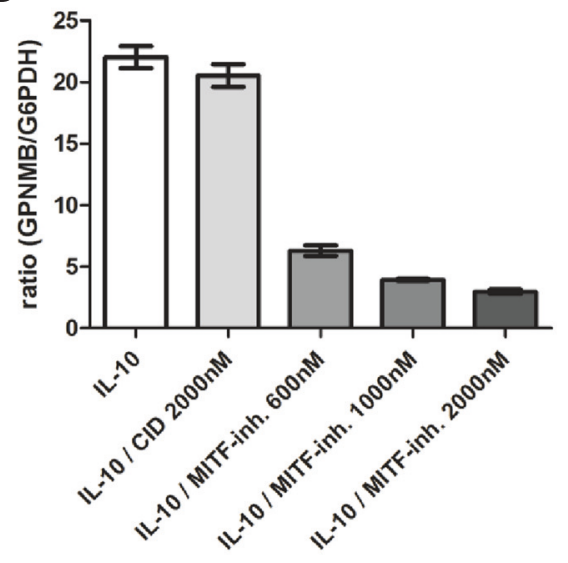

C

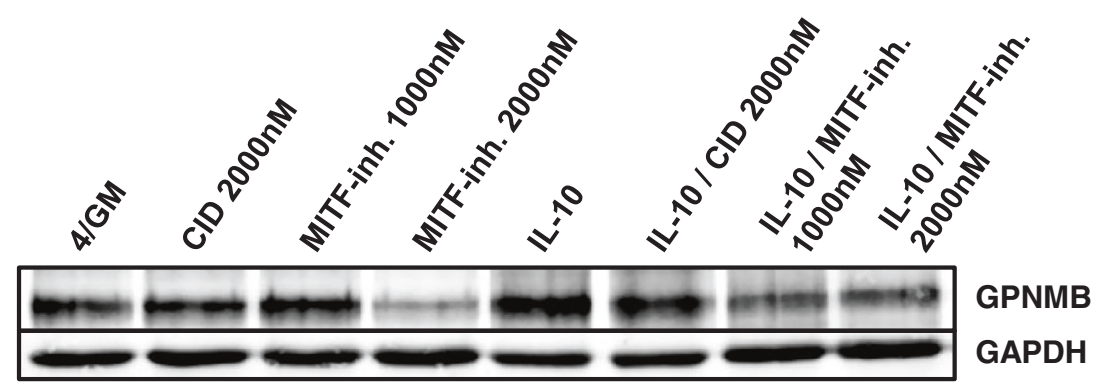

Figure 6 MITF-Inhibition decreases GPNMB gene expression in moDC. moDC were generated in vitro with GM-CSF, IL-4 and DMSO alone (4/GM) with or without IL-10 and additional MITF inhibitor ML329 (MITF-inh.; 600 nM - 2000 nM) or KLF5 expression inhibitor CID (2000 nM) as control and analyzed for GPNMB expression. (A, B) qRT-PCR analysis: relative level of GPNMB mRNA. The mean $( \pm S D)$ of duplicate measurements is shown. (C) GPNMB protein levels were analyzed by western blotting. GAPDH served as loading control. Exemplary results from at least three independent experiments using different donors are presented. 
or without IL-10 and treated with $1000 \mathrm{nM}$ and $2000 \mathrm{nM}$ ML329 (Figure 6C). Moreover, decrease of cell surface protein on moDC generated with imatinib or nilotinib and treated with $2000 \mathrm{nM}$ ML329 was seen in FACS analyses (Additional file 6: Figure S6).

In summary, these experiments demonstrate that GPNMB expression in moDC is mediated by the transcription factor MITF as endpoint of the (inhibited) PI3K/Akt pathway.

Inhibition of the PI3K/Akt pathway results in reduction of moDC T cell stimulatory capacity that is restored by soluble SD-4

We have previously shown that GPNMB upregulation upon exposure to imatinib, dasatinib or nilotinib results in significantly reduced $\mathrm{T}$ cell stimulatory capacity of moDC [20]. To confirm the functional relevance of Akt inhibition, we performed $\mathrm{T}$ cell proliferation (MLR) assays. For this reason, immature moDC exposed to MK2206 or imatinib during development were cultured with allogeneic peripheral blood mononuclear cells (PBMC) for 5 days and then incubated with ${ }^{3} \mathrm{H}$-thymidine. Treatment with Akt inhibitor or imatinib resulted in significantly reduced capacity to stimulate proliferation of allogeneic $\mathrm{T}$ cells to an average of $\sim 50 \%$ or $\sim 60 \%$, respectively. (Figure 7 and Additional file 7: Figure S7). Chung et al. previously reported SD-4 to be the T cell ligand through which GPNMB mediates its negative coregulatory function [15-18]. Therefore, we assessed the specific role of GPNMB upregulation upon MK2206 treatment by addition of recombinant ligand SD-4. As demonstrated in Figure 7, blockade of endogenous SD-4 by addition of increasing amounts of soluble SD-4 restored the capacity of MK2206 treated moDC to stimulate proliferation of T cells, while an irrelevant recombinant protein (Klotho $\beta$ ) had no effect (Figure 7 and Additional file 7: Figure S7).

These experiments confirm that targeted inhibition of Akt signaling tunes the development of primary moDC towards a suppressive phenotype and demonstrate that upregulation of GPNMB is critically involved in the inhibition of DC function.

\section{Discussion}

Ex vivo generated and manipulated immunogenic DC are used in anti-cancer vaccines in experimental and clinical studies [35]. On the other hand, so-called tolerogenic DC are considered an interesting alternative to conventional immunosuppressive therapies since it has been proven in animal models that their application prevents rejection of transplanted organs. Currently, tolerogenic DC are also tested in patients with GvHD and in animal models of autoimmune diseases [36-41].

The in vitro generation of DC from two specific precursor populations is well established: monocytes and CD34 ${ }^{+}$ stem cells. However, the usage of primary moDC is advantageous as they are simple to generate in adequate numbers and capable of inducing potent specific $\mathrm{T}$ cell responses $[42,43]$. Adoptive immunotherapy using this cell type for cancer treatment induces remarkable response rates and in clinical studies has shown to improve survival even in patients suffering from advanced disease [44-48].

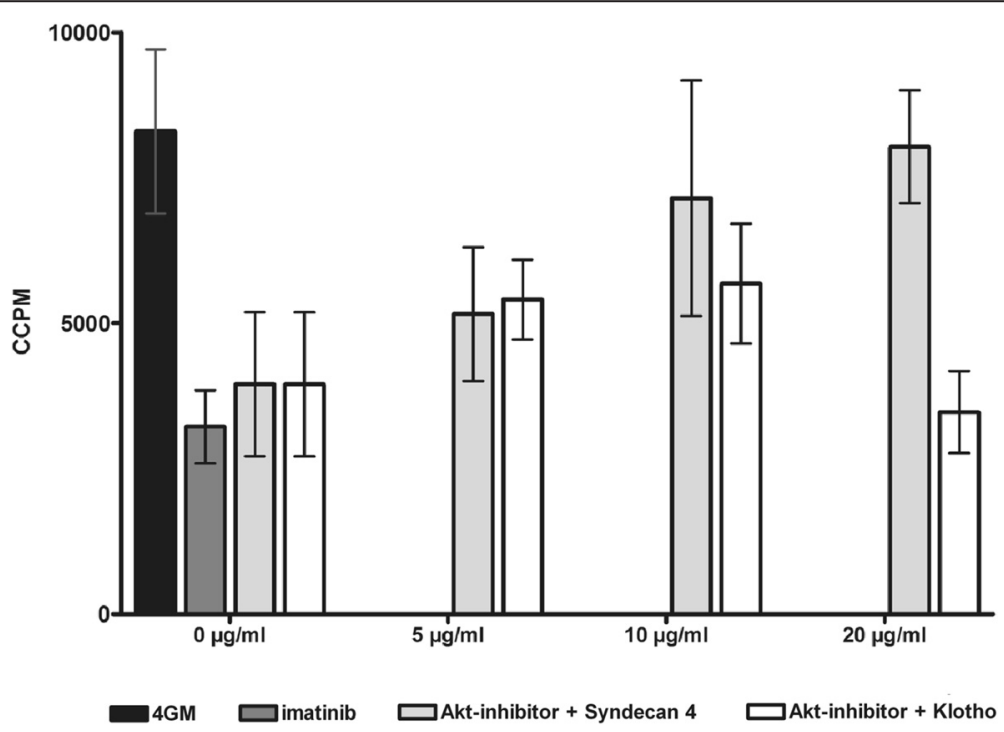

Figure 7 Akt inhibition reduces the capacity of human moDC to induce $T$ cell responses. moDC generated in vitro with GM-CSF and IL-4 alone (4/GM) or with imatinib (3 $\mu \mathrm{M})$ or Akt inhibitor MK2206 (300 nM) were used as stimulators in MLR with allogeneic T cells. Increasing concentration $(0.0 \mu \mathrm{g} / \mathrm{mL}-20 \mu \mathrm{g} / \mathrm{mL})$ of blocking soluble recombinant T cell ligand SD-4 were added with recombinant Klotho $\beta$ serving as control. T cell proliferation was measured by $\left[^{3} \mathrm{H}\right]$ thymidine incorporation. CCPM $=$ corrected counts per minute. The mean $( \pm \mathrm{SD})$ of quadruple measurements is shown. Exemplary result from three independent experiments using different donors is presented. 
Fortunately, in the last decade development of novel targeted therapies have significantly improved treatment of cancer. One fascinating example is the introduction of BCR-ABL TKI to CML treatment. However, discontinuation of imatinib results in a relapse rate in about $50 \%$ of patients [49]. Combination of targeted therapy and immunotherapy, with the goal to eradicate minimal residual disease is therefore moving into the focus of interest. Essential for the application of such combinatory approaches certainly is to analyze the influence of the respective TKI on cellular signal transduction in immune cells.

Depending on the micro milieu, DC can be found in distinct differentiation stages: immature, mature or semimature. Numerous factors induce and/or regulate DC maturation, of which TLR ligands are the most prominent. TLR signaling leads to phosphorylation of inhibitory IKB proteins by activated IKK and the subsequent release of NF- $\mathrm{kB}$ transcription factors, which translocate to the nucleus to induce expression of pro-inflammatory target genes [50]. Ozes et al. have demonstrated that Akt, activated by TNF, mediates IKK phosphorylation and subsequent NF- $\kappa B$ activation [51]. Simultaneously, activated Akt inhibits GSK3 $\beta$ by phosphorylation at serine 9 . It was proposed, if Akt is inactive, that the p105 precursor of the NF-KB p50 subunit is stabilized through phosphorylation by GSK3 $\beta$, thus preventing formation of functional active NF-kB [52].

Previously, we have established that exposure of human $\mathrm{CD} 14^{+}$peripheral blood monocytes to therapeutic concentrations of imatinib during differentiation into moDC affects their phenotype, cytokine secretion, and $\mathrm{T}$ cell stimulatory capacity due to inhibition of NF- $\mathrm{kB}$ and Akt signaling pathways [27]. Furthermore, we have demonstrated that IL-10 prevents nuclear translocation, DNA binding and TLR-induced nuclear expression of the NF- $\mathrm{kB}$ family members c-Rel and Rel-B as well as IRF-3 and IRF-8 as a result of inhibitory effects on the PI3K pathway $[19,53]$.

In the present study we show for the first time that the concordant inhibition of the PI3K/Akt signaling pathway by the clinically used BCR-ABL TKI imatinib or nilotinib, Akt inhibitor MK2206 or the immunosuppressive cytokine IL-10 activates the downstream serine/threonine protein kinase GSK3ß, and subsequently the transcription factor MITF that is translocated into the cell nucleus. Moreover, treatment of moDC with the small molecule inhibitor of the MITF molecular pathway ML329 [22] reduced the expression of GPNMB at the level of mRNA and protein, indicating that GPNMB expression is in fact facilitated by MITF activation.

Based on these and previous results, we suggest the following general model for balancing activating and inhibitory signals in primary moDC: the central regulatory molecules in signal processing are the serine/threonine kinases Akt and GSK3ß. Activating signals such as growth factors or TLR stimulation lead to phosphorylation of Akt that causes phosphorylation and thereby inhibition of GSK3 $\beta$. Inactive GSK3 $\beta$ permits the formation of functional members of the NF- $\mathrm{kB}$ transcription factor family that induce the transcription of proinflammatory genes. By contrast, inhibitory stimuli, such as the antiinflammatory cytokine IL-10 or BCR-ABL TKI, which suppress Akt signaling, lead to activation of GSK3 $\beta$ and the transcription factor MITF that drives expression of inhibitory molecules such as GPNMB.

Lundberg et al. recently analysed the gene expression profiles of commonly used in vitro DC models (moDC, CD $34^{+}$-derived Langerhans cells, CD $34^{+}$-derived DC and MUTZ-3 DC) and found MITF to be expressed $>2$ fold higher in moDC as compared to each of the other in vitro generated DC [54]. These results and own expression analyses (Figure 4) support our model in which MITF is a relevant transcription factor in moDC. However, a function of MITF for signal transduction in human peripheral blood DC has still to be elucidated.

A number of studies have also shown the direct influence of imatinib on $\mathrm{T}$ lymphocytes in vitro. Cwynarski et al. found that this TKI inhibited T cell proliferation and reduced the production of IFN- $\gamma$ [55]. Similar results were obtained by Dietz et al.: imatinib inhibited $\mathrm{T}$ cell proliferation induced by allogeneic DC [56]. A further study found the expression of the activation markers CD25 and CD69 as well as secretion of IL-2 to be suppressed in activated $\mathrm{T}$ cells [57]. Taken together, the direct effects of imatinib on T cells, as well as its indirect, mediated via $\mathrm{DC}$, point to the same direction: the inhibition of $\mathrm{T}$ cell function. However, the specific contribution of TKI treated DC in vivo still has to be proven and elaborated.

Our research provides an important basis for the in vitro manipulation of moDC to induce overexpression of GPNMB for the treatment of exaggerated immune responses. Our results might also be relevant in another context: GPNMB is expressed at higher levels in melanoma and breast cancer [58]. The anti-GPNMB antibodydrug conjugate CR011-vcMMAE (glembatumumab vedotin) thus was tested for the treatment of these tumors in phase I/II-clinical studies [59-61]. In this context, attempts were made to increase GPNMB expression in cell lines by treatment with various therapeutics to enhance the binding of CR011-vcMMAE. Interestingly, imatinib, as described here for primary moDC, induced GPNMB expression in melanoma and glioblastoma cell lines. However, the signaling mechanism was not elucidated [62]. It remains an interesting task to investigate the expression and function of GPNMB in other tumor entities in two respects: On the one hand GPNMB is a potential tumorassociated antigen that could be an attractive target for 
immunotherapeutic approaches. On the other hand GPNMB represents a molecule that suppresses $\mathrm{T}$ cell responses and permits tumor escape. For both aspects, specific manipulation of GPNMB expression could be of clinical use for the development of novel treatment approaches for malignant and autoimmune disease.

\section{Conclusions}

The results of the present study demonstrate that the immunosuppressive cytokine IL-10 and the therapeutically used BCR-ABL TKI imatinib or nilotinib, examined here, concordantly lead to dephosphorylation and thereby activation of the serine/threonine protein kinase GSK3ß via inhibition of PI3K/Akt signaling in human moDC. This leads to phosphorylation and translocation of MITF to the nucleus. MITF is a transcription factor whose function in hematopoietic and blood cells was unknown so far. Using a small molecule inhibitor of MITF activity we confirmed that MITF is a direct positive regulator of GPNMB expression in moDC. Moreover, treatment with BCR-ABL TKI or PI3K/Akt inhibitors resulted in profound upregulation of GPNMB that resulted in reduced stimulatory capacity of moDC in MLR with allogenic $T$ cells. This impairment could be restored by addition of the GPNMB $\mathrm{T}$ cell ligand SD-4.

Our data extend the current understanding regarding the molecular mechanisms that balance activating and inhibitory signals in DC. Manipulation of the involved signaling cascades and in particular GPNMB expression/ function may constitute a promising strategy in combinatory approaches using BCR-ABL TKI and DC-based immunotherapy and may also allow for manipulation of $\mathrm{T}$ cell responses in GvHD.

\section{Methods}

Generation of monocyte-derived dendritic cells (moDC) moDC were generated ex vivo from $\mathrm{CD} 14^{+}$peripheral blood primary monocytes that were either purified by magnetic cell sorting (CD14 MicroBeads, Miltenyi, Bergisch Gladbach, Germany) or plastic adherence. Peripheral blood mononuclear cells (PBMC) were isolated by Ficoll/Paque (Biochrom, Berlin, Germany) density gradient centrifugation of buffy coats obtained from healthy volunteers (Blood Donation Center, University of Tübingen). For plastic adherence cells were seeded $\left(1 \times 10^{8} / 10 \mathrm{~mL}\right)$ into $75 \mathrm{~cm}^{2}$ cell culture flasks (Corning, Cambridge, MA, USA) in serumfree X-VIVO 20 medium (Cambrex Bio Science, Verviers, Belgium). After $2 \mathrm{~h}$ of incubation at $37^{\circ} \mathrm{C} / 5 \% \mathrm{CO}_{2}$, non-adherent cells were removed. The monocytes were cultured in $10 \mathrm{~mL}$ RP10 medium (RPMI 1640 with glutamax-I, supplemented with $10 \%$ inactivated fetal calf serum, and antibiotics (Invitrogen, Karlsruhe, Germany)) supplemented with granulocyte macrophage colonystimulating factor (GM-CSF, $100 \mathrm{ng} / \mathrm{mL}$; Leukine Liquid
Sargramostim, Sanofi, Bridgewater, USA) and IL-4 (20 ng/mL; R\&D Systems, Wiesbaden, Germany) added every $2^{\text {nd }}$ day for 7 days. IL-10 (10 ng/mL; R\&D Systems), imatinib (3 $\mu \mathrm{M}$; Cayman, Biomol, Hamburg, Germany), nilotinib (3 $\mu$ M; Cayman), Akt inhibitor MK2206 (300 nM; Selleckchem, München, Germany), Erk inhibitor FR180204 (300 nM; Calbiochem, Merck Millipore, Darmstadt, Germany), PI3K inhibitor LY294002 (100 nM; Cayla InvivoGen, Toulouse, France), c-Raf inhibitor 553003 (20 nM; Calbiochem)), MITF inhibitor ML329 (200 nM $2000 \mathrm{nM}$ in dimethyl sulfoxide (DMSO); Glixx Laboratories, Southborough, MA, USA) or Kruppel-like factor 5 (KLF5) expression inhibitor CID-5951923 (2000 nM in DMSO; Glixx Laboratories) were added starting from day 0 of cell culture every $2^{\text {nd }}$ day where indicated. Maturation was induced on day 6 by adding LPS (TLR4L, $100 \mathrm{ng} / \mathrm{mL}$; Sigma-Aldrich, Deisenhofen, Germany) where indicated. After 7 days of culture, if necessary, DC-SIGN ${ }^{+}\left(\mathrm{CD} 209^{+}\right)$ moDC were enriched to $>90 \%$ purity prior to $\mathrm{qRT}-\mathrm{PCR}$ and western blot analyses (CD209 MicroBead Kit, Miltenyi). Prior to lysis, purified cells were further incubated for 20, 40, 60 or 90 min under the conditions that were applied at the beginning of culture.

\section{Quantitative reverse transcriptase PCR (qRT-PCR)}

Quantification of GPNMB gene transcripts was conducted using a LightCycler carousel-based system (Roche, Mannheim, Germany) as described previously [20]. MITF transcripts (all isoforms) were quantified with primers 5'ggagcttccaaaacaagcag-3', 5'-acaagtgtgctccgtctcttc-3' and Universal ProbeLibrary probe \#68 (Roche). The relative mRNA levels were calculated as the ratio target gene/ G6PDH. For the analysis of hematopoietic and blood cells, cell-type specific total RNA (Miltenyi) was used.

\section{Immunostaining}

moDC were stained using FITC-, PE-, PerCP-Cy5.5 or allophycocyanin (APC) conjugated mouse monoclonal antibodies against CD1a, CD86, CD14 (PharMingen, Hamburg, Germany), CD80, CD83, HLA-DR, (Becton Dickinson, Heidelberg, Germany), CD86 (PharMingen), DC-SIGN (CD209; eBioscience, Frankfurt, Germany), and mouse IgG isotype control (Becton Dickinson, eBioscience). GPNMB was detected using an antihuman GPNMB antibody (R\&D Systems) conjugated to PE (Lightning-Link R-PE conjugation kit, Innova Bioscience, Cambrige, UK).

To exclude dead cells a viability dye (Fixable Viability Dye eFluor 660; eBioscience) was included in all analyses. For detection of apoptosis, the Annexin V-Fluos Staining kit (Roche) was used according to the instructions of the manufacturer. Analyses were performed on a FACSCalibur cytometer (Becton Dickinson). Data were analyzed using Flowjo software. The values have been 
calculated as follows: DFMI $=$ median fluorescence intensity of $\mathrm{CD} 209^{+}$cells - median isotype control fluorescence intensity of $\mathrm{CD}^{2} \mathrm{9}^{+}$cells. Histogram overlays are displayed as \%Max, scaling each curve to mode $=100 \%$.

\section{Western blotting}

If necessary, prior to lysis, positive selection of DC-SIGN (CD209) expressing cells was conducted using the CD209 (DC-SIGN) MicroBead Kit (Miltenyi). Whole cell lysates were prepared from moDC as described previously [20]. Separation of nuclear extract from the cytoplasmic fraction was performed using the Nuclear/Cytosol Fractionation Kit (BioVision; BioCat, Heidelberg, Germany). To prevent proteolytic degradation during cell lysis, Halt Protease and Phosphatase Inhibitor Cocktail (Fisher Scientific, Schwerte, Germany) was added to the lysis buffer. The protein levels of Akt-(P), GSK3 $\alpha / \beta-(\mathrm{P})$ and MITF were determined by separating 10-30 $\mu \mathrm{g}$ whole cell lysates, nuclear or cytoplasmic protein fractions on a $10.5 \%$ or $12.0 \%$ SDS-polyacrylamide gel and subsequent transfer of protein to nitrocellulose membranes (Whatman, Dassel, Germany). The blots were probed with the following primary antibodies: monoclonal mouse anti-human Akt (R\&D Systems), monoclonal rabbit anti-human PhosphoAkt (Ser473), monoclonal rabbit anti-human GSK3 $\alpha / \beta$, polyclonal rabbit anti-human Phospho-GSK3 $\alpha / \beta$ (Ser 21/ 9) (Cell Signaling, Frankfurt, Germany), polyclonal rabbit anti-human MITF (abcam, Cambridge, UK) and polyclonal rabbit-anti-GAPDH (Merck Millipore, Darmstadt, Germany) or monoclonal mouse-anti-GAPDH (R\&D Systems). Corresponding secondary antibodies were purchased from LI-COR Biotechnology (Bad Homburg, Germany): IRDye 680 Donkey anti-rabbit IgG $(\mathrm{H}+\mathrm{L})$, IRDye 680RD Donkey anti-mouse IgG $(\mathrm{H}+\mathrm{L})$, IRDye 800CW Donkey anti-rabbit IgG $(\mathrm{H}+\mathrm{L})$ and IRDye 800CW Donkey anti-rabbit IgG $(\mathrm{H}+\mathrm{L})$. Different antigens were detected simultaneously on the same blot using IRDye secondary antibodies labeled with spectrally distinct fluorescent dyes. The Odyssey Infrared Imaging System (LI-COR Biotechnology) was used for western blot analysis.

\section{Mixed lymphocyte reaction (MLR)}

moDC were inactivated by $\gamma$-radiation at $30 \mathrm{~Gy}, 100 \%$, and were seeded into 96-well microplates (Greiner Bio-One, Frickenhausen, Germany) at concentrations of $1 \times 10^{4}$ cells/well. Blocking recombinant ligand SD-4 (R\&D Systems) was added with recombinant Klotho $\beta$ (R\&D Systems) serving as control. A total of $1 \times 10^{5}$ responding cells from freshly isolated allogeneic PBMC were added to the previously prepared $1 \times 10^{4}$ stimulator cells $(\mathrm{moDC})$. Thymidine incorporation was measured on day 5 by a $16 \mathrm{~h}$ pulse with $\left[{ }^{3} \mathrm{H}\right]$ thymidine $(0.5 \mu \mathrm{Ci}$ [0.0185 MBq]/well; GE Health- care, Munich, Germany).

\section{Availability of supporting data}

The data sets supporting the results of this article are included within the article and its additional files.

\section{Additional files}

Additional file 1: Figure S1. PI3K/Akt-inhibition upregulates GPNMB mRNA levels in human moDC. Combined analysis of different donors. Immature moDC were generated in vitro with GM-CSF and IL-4 alone (4/GM) or with additional Akt inhibitor MK2206 (300 nM) or Erk inhibitor FR180204 (300 nM) and analyzed for GPNMB mRNA expression by qRT-PCR. The relative level of GPNMB mRNA in a sample was expressed as the ratio GPNMB/G6PDH. The values were normalized to $100 \%$ for IL-4 and GM-CSF treated $\mathrm{moDC}$. Stars indicate significance ${ }^{* *} \mathrm{P}<.01$, n.s. $=$ not significant; Wilcoxon matched-pairs signed rank test).

Additional file 2: Figure S2. Imatinib, nilotinib, Akt inhibitor or IL-10 inhibit phosphorylation of Akt in human moDC. Combined analysis of different donors. moDC were generated in vitro with GM-CSF and IL-4 alone (4/GM) or with additional imatinib $(3 \mu \mathrm{M})$, nilotinib $(3 \mu \mathrm{M})$, Akt inhibitor MK2206 (300 $\mathrm{nM})$ or IL-10 (10 ng/mL) and analyzed by western blotting. The relative level of phosphorylated Akt protein (Akt-P) in a sample was expressed as the ratio Akt-P/GAPDH (loading control). Quantitative analysis was performed using the LI-COR Odyssey Application Software 3.0. The values were normalized to $100 \%$ for IL-4 and GM-CSF treated moDC. The mean ( \pm SD) obtained from measurements of different donors is shown. The raw data were used to perform Student's t-test (ratio paired, two-sided, equal variance). Stars indicate significance $\left({ }^{*} \mathrm{P}<.01,{ }^{* *} \mathrm{P}<.003, \mathrm{n} . \mathrm{s} .=\right.$ not significant).

Additional file 3: Figure S3. Imatinib, nilotonib, Akt inhibitor or IL-10 prevent phosphorylation of GSK3ß in human moDC. Combined analysis of different donors. moDC were generated in vitro with GM-CSF and IL-4 alone $(4 / \mathrm{GM})$ or with additional imatinib $(3 \mu \mathrm{M})$, nilotinib $(3 \mu \mathrm{M})$, Akt inhibitor MK2206 (300 nM) or IL-10 (10 ng/mL) and analyzed by western blotting. The relative level of phosphorylated GSK3ß protein (GSK3ß-P) in a sample was expressed as the ratio GSK3ß-P/GAPDH (loading control). Quantitative analysis was performed using the LI-COR Odyssey Application Software 3.0. The values were normalized to $100 \%$ for IL-4 and GM-CSF treated moDC. The mean $( \pm S D)$ obtained from measurements of different donors is shown. The raw data were used to perform Student's $t$-test (ratio paired, two-sided, equal variance). Stars indicate significance $\left({ }^{*} P<.05\right.$, ${ }^{* *} P<.01$, ${ }^{* *} P<.003$, n.s. = not significant).

Additional file 4: Figure S4. MITF transcript levels are increased upon treatment with imatinib, nilotinib or IL-10. Combined analysis of different donors. Immature moDC were generated in vitro with GM-CSF and IL-4 alone (4/GM) or with additional TKI ( $3 \mu \mathrm{M}$ imatinib or $3 \mu \mathrm{M}$ nilotinib) or IL-10 and analyzed for MITF mRNA expression by qRT-PCR. The relative level of MITF mRNA in a sample was expressed as the ratio MITF/G6PDH. The values were normalized to $100 \%$ for IL-4 and GM-CSF treated moDC.

Additional file 5: Figure S5. MITF-Inhibition downregulates GPNMB mRNA expression in moDC. Combined analysis of different donors. Analysis of GPNMB mRNA levels by qRT-PCR. moDC were generated in vitro with (A) GM-CSF, IL-4 and DMSO alone (4/GM) or with additional MITF inhibitor ML329 (MITF-inh.; 200 nM - 2000 nM) or KLF5 expression inhibitor CID (2000 nM) as control. (B) moDC were generated with additional IL-10 alone or together with MITF inhibitor ML329 (IL-10; MITF-inh.; 200 nM - 2000 $\mathrm{nM}$ ) or KLF5 expression inhibitor CID (IL-10; CID $2000 \mathrm{nM})$ as control. The relative level of GPNMB mRNA in a sample was expressed as the ratio GPNMB/G6PDH. The values were normalized to $100 \%$ for DMSO or DMSO/IL-10 treated moDC. The raw data were used to perform Student's $t$-test (ratio paired, two-sided, equal variance). Stars indicate significance ( ${ }^{*} \mathrm{P}<.05$, ${ }^{* *} \mathrm{P}<.01,{ }^{* * *} \mathrm{P}<.003$, n.s. $=$ not significant).

Additional file 6: Figure S6. MITF-Inhibition decreases GPNMB cell surface protein on moDC generated with imatinib or nilotinib. moDC were generated in vitro with GM-CSF, IL-4 and imatinib or nilotinib alone and additional MITF inhibitor ML329 (MITF-inh.; 2000 nM) or KLF5 expression inhibitor CID (2000 $\mathrm{nM}$ ) as control. GPNMB protein level of $\mathrm{CD}_{209^{+}}$moDC was analyzed by flow cytometry. Data were analyzed using FlowJo software and histogram overlays are displayed as \%Max, scaling each curve to mode $=100 \%$. 
Additional file 7: Figure S7. Akt inhibition reduces the capacity of human moDC to induce $T$ cell responses. Combined analysis of different donors. moDC generated in vitro with GM-CSF and IL-4 alone (4/GM) or with imatinib $(3 \mu \mathrm{M})$ or Akt inhibitor MK2206 (Akt.-inh., 300 nM) were used as stimulators in MLR with allogeneic T cells. T cell proliferation was measured by $\left[{ }^{3} H\right]$ thymidine incorporation. CCPM $=$ corrected counts per minute. (A) Combined analysis of 5 different donors. The values were normalized to $100 \%$ for IL-4 and GM-CSF treated moDC. (B) Increasing concentration $(0.0 \mu \mathrm{g} / \mathrm{mL}-20.0 \mu \mathrm{g} / \mathrm{mL})$ of blocking soluble recombinant T cell ligand SD-4 were added with recombinant Klotho $\beta$ serving as control. Combined analyses of 3 different donors. The values were normalized to $100 \%$ for Akt inhibitor MK2206 (Akt.-inh., 300 nM) treated moDC. The raw data were used to perform Student's $t$-test (ratio paired, two-sided, equal variance). Stars indicate significance ${ }^{*} \mathrm{P}<.05$, n.s. $=$ not significant; absolute data were analyzed using Student's t-test (ratio paired, one-sided, equal variance).

\section{Abbreviations}

APC: Antigen-presenting cells; CML: Chronic myeloid leukemia; DC: Dendritic cell(s); DC-SIGN: DC-specific ICAM-3 grabbing nonintegrin; DMSO: Dimethyl sulfoxide; FACS: Flow Cytometry; FITC: Fluorescein isothiocyanate; GMCSF: Granulocyte macrophage colony-stimulating factor; GPNMB: Glycoprotein NMB; GSK3: Glycogen synthase kinase-3; GvHD: Graft-versus-host disease; IKK: IKB kinase; LPS: Lipopolysaccharide; MITF: Microphthalmia-associated transcription factor; MLR: Mixed lymphocyte reactions; moDC: monocyte-derived dendritic cells; NF-KB: nuclear factor-KB; PBMC: Peripheral blood mononuclear cells; PDGF: Platelet-derived growth factor; PE: Phycoerythrin; PerCP: Peridinin-chlorophyll proteins; PI3K: Phosphatidylinositide 3-kinase; qRT-PCR: Quantitative reverse transcriptase PCR; SD-4: Syndecan-4; TKI: Tyrosine kinase inhibitors; TLR: Toll-Like Receptor.

\section{Competing interests}

The authors declare that they have no competing interests.

\section{Authors' contributions}

$M G, J G, S J, F G$ and SMR performed experiments, analyzed results, and made the figures; MG, DD, HRS, MRM, FG and SMR designed the research, discussed results, wrote and edited the paper. All authors read and approved the final manuscript.

\section{Acknowledgments supported by the European Social Fund in Baden-Württemberg. We technical assistance. \\ Received: 6 August 2014 Accepted: 10 March 2015 \\ Published online: 24 March 2015}

This work was supported by Deutsche Krebshilfe (project no. 109046). SMR is acknowledge support by Deutsche Forschungsgemeinschaft and Open Access Publishing Fund of University of Tübingen. We thank Sylvia Klein for excellent

\section{References}

1. Driessens G, Kline J, Gajewski TF. Costimulatory and coinhibitory receptors in anti-tumor immunity. Immunol Rev. 2009;229:126-44.

2. Nurieva RI, Liu X, Dong C. Molecular mechanisms of T-cell tolerance. Immunol Rev. 2011;241:133-44

3. Pardoll DM. The blockade of immune checkpoints in cancer immunotherapy. Nat Rev Cancer. 2012;12:252-64.

4. Manicassamy S, Pulendran B. Dendritic cell control of tolerogenic responses. Immunol Rev. 2011;241:206-27.

5. Ballestrero A, Boy D, Moran E, Cirmena G, Brossart P, Nencioni A. Immunotherapy with dendritic cells for cancer. Adv Drug Deliv Rev. 2008;60:173-83.

6. Nencioni A, Grünebach F, Schmidt SM, Müller MR, Boy D, Patrone F, et al. The use of dendritic cells in cancer immunotherapy. Crit Rev Oncol Hematol. 2008:65:191-9.

7. Vacchelli E, Vitale I, Eggermont A, Fridman WH, Fucikova J, Cremer I, et al. Trial watch: Dendritic cell-based interventions for cancer therapy. Oncoimmunology. 2013;2:e25771.
8. Buchdunger E, Zimmermann J, Mett H, Meyer T, Muller M, Druker BJ, et al. Inhibition of the Abl protein-tyrosine kinase in vitro and in vivo by a 2-phenylaminopyrimidine derivative. Cancer Res. 1996;56:100-4.

9. Druker BJ, Tamura S, Buchdunger E, Ohno S, Segal GM, Fanning S, et al. Effects of a selective inhibitor of the Abl tyrosine kinase on the growth of Bcr-Abl positive cells. Nat Med. 1996;2:561-6.

10. Druker BJ, Sawyers CL, Kantarjian H, Resta DJ, Reese SF, Ford JM, et al. Activity of a specific inhibitor of the BCR-ABL tyrosine kinase in the blast crisis of chronic myeloid leukemia and acute lymphoblastic leukemia with the Philadelphia chromosome. N Engl J Med. 2001;344:1038-42.

11. Kantarjian H, Shah NP, Hochhaus A, Cortes J, Shah S, Ayala M, et al. Dasatinib versus Imatinib in Newly Diagnosed Chronic-Phase Chronic Myeloid Leukemia. N Engl J Med. 2010;362:2260-70.

12. Saglio G, Kim DW, Issaragrisil S, le Coutre P, Etienne G, Lobo C, et al. Nilotinib versus Imatinib for Newly Diagnosed Chronic Myeloid Leukemia. N Engl J Med. 2010;362:2251-9.

13. Saglio G, Hochhaus A, Goh YT, Masszi T, Pasquini R, Maloisel F, et al. Dasatinib in Imatinib-Resistant or Imatinib-Intolerant Chronic Myeloid Leukemia in Blast Phase After 2 Years of Follow-Up in a Phase 3 Study Efficacy and Tolerability of 140 Milligrams Once Daily and 70 Milligrams Twice Daily. Cancer. 2010;116:3852-61.

14. Chung JS, Sato K, Dougherty II, Cruz Jr PD, Ariizumi K. DC-HIL is a negative regulator of T lymphocyte activation. Blood. 2007;109:4320-7.

15. Chung JS, Dougherty I, Cruz PD, Ariizumi K. Syndecan-4 mediates the coinhibitory function of DC-HIL on T cell activation. J Immunol. 2007;179:5778-84.

16. Chung JS, Bonkobara M, Tomihari M, Cruz PA, Ariizumi K. The DC-HIL/syndecan-4 pathway inhibits human allogeneic T-cell responses. Eur J Immunol. 2009:39:965-74.

17. Chung JS, Cruz Jr PD, Ariizumi K. Inhibition of T-cell activation by syndecan-4 is mediated by CD148 through protein tyrosine phosphatase activity. Eur J Immunol. 2011:41:1794-9.

18. Chung JS, Tomihari M, Tamura K, Kojima T, Cruz Jr PD, Ariizumi K. The DC-HIL ligand syndecan- 4 is a negative regulator of T-cell allo-reactivity responsible for graft-versus-host disease. Immunology. 2013;138:173-82.

19. Knödler A, Schmidt SM, Bringmann A, Weck MM, Brauer KM, Holderried TA, et al. Post-transcriptional regulation of adapter molecules by IL-10 inhibits TLR-mediated activation of antigen-presenting cells. Leukemia. 2009;23:535-44.

20. Schwarzbich MA, Gutknecht M, Salih J, Salih HR, Brossart P, Rittig SM, et al. The immune inhibitory receptor osteoactivin is upregulated in monocyte-derived dendritic cells by BCR-ABL tyrosine kinase inhibitors. Cancer Immunol Immunother. 2012;61:193-202.

21. Shibahara S, Takeda K, Yasumoto K, Udono T, Watanabe K, Saito H, et al. Microphthalmia-associated transcription factor (MITF): multiplicity in structure, function, and regulation. J Investig Dermatol Symp Proc. 2001;6:99-104.

22. Faloon PW, Bennion M, Weiner WS, Smith RA, Wurst J, Weiwer M, Hartland C, Mosher CM, Johnston S, Porubsky P, Neuenswander B, Dandapani S, Munoz B Schoenen FJ, Metkar S, Haq R, Fisher DE, Aube J, Palmer M, Schreiber SL. A Small Molecule Inhibitor of the MITF Molecular Pathway. Probe Reports from the NIH Molecular Libraries Program. Bethesda (MD): National Center for Biotechnology Information (US) 2013.

23. Wang Y, Radfar S, Liu S, Riker Al, Khong HT. Mitf-Mdel, a novel melanocyte/ melanoma-specific isoform of microphthalmia-associated transcription factor-M, as a candidate biomarker for melanoma. BMC Med. 2010;8:14.

24. Hantschel O, Rix U, Superti-Furga G. Target spectrum of the BCR-ABL inhibitors imatinib, nilotinib and dasatinib. Leukemia \& Lymphoma. 2008;49:615-9.

25. Bhattacharyya S, Sen P, Wallet M, Long B, Baldwin Jr AS, Tisch R. Immunoregulation of dendritic cells by IL-10 is mediated through suppression of the PI3K/Akt pathway and of IkappaB kinase activity. Blood. 2004;104:1100-9.

26. Laird MH, Rhee SH, Perkins DJ, Medvedev AE, Piao W, Fenton MJ, et al. TLR4/ MyD88/PI3K interactions regulate TLR4 signaling. J Leukoc Biol. 2009;85:966-77.

27. Appel S, Rupf A, Weck MM, Schoor O, Brümmendorf TH, Weinschenk T, et al. Effects of imatinib on monocyte-derived dendritic cells are mediated by inhibition of nuclear factor-kappaB and Akt signaling pathways. Clin Cancer Res. 2005;11:1928-40.

28. Xie J, Qian J, Yang J, Wang S, Freeman III ME, Yi Q. Critical roles of Raf/MEK/ERK and PI3K/AKT signaling and inactivation of p38 MAP kinase in the differentiation and survival of monocyte-derived immature dendritic cells. Exp Hematol. 2005;33:564-72.

29. Cross DA, Alessi DR, Cohen P, Andjelkovich M, Hemmings BA. Inhibition of glycogen synthase kinase- 3 by insulin mediated by protein kinase B. Nature. 1995;378:785-9. 
30. Fang X, Yu SX, Lu Y, Bast Jr RC, Woodgett JR, Mills GB. Phosphorylation and inactivation of glycogen synthase kinase 3 by protein kinase $A$. Proc Natl Acad Sci U S A. 2000;97:11960-5.

31. Ripoll VM, Meadows NA, Raggatt LJ, Chang MK, Pettit AR, Cassady Al, et al. Microphthalmia transcription factor regulates the expression of the novel osteoclast factor GPNMB. Gene. 2008;413:32-41.

32. Terragni J, Nayak G, Banerjee S, Medrano JL, Graham JR, Brennan JF, et al. The E-box binding factors Max/Mnt, MITF, and USF1 act coordinately with FoxO to regulate expression of proapoptotic and cell cycle control genes by phosphatidylinositol 3-kinase/Akt/glycogen synthase kinase 3 signaling. J Biol Chem. 2011;286:36215-27.

33. Takeda K, Takemoto C, Kobayashi I, Watanabe A, Nobukuni Y, Fisher DE, et al. Ser298 of MITF, a mutation site in Waardenburg syndrome type 2, is a phosphorylation site with functional significance. Hum Mol Genet. 2000;9:125-32.

34. Bronisz A, Sharma SM, Hu R, Godlewski J, Tzivion G, Mansky KC, et al. Microphthalmia-associated transcription factor interactions with 14-3-3 modulate differentiation of committed myeloid precursors. Mol Biol Cell. 2006;17:3897-906

35. Palucka K, Banchereau J. Cancer immunotherapy via dendritic cells. Nat Rev Cancer. 2012;12:265-77.

36. Hackstein H, Thomson AW. Dendritic cells: emerging pharmacological targets of immunosuppressive drugs. Nat Rev Immunol. 2004;4:24-34.

37. Horibe EK, Sacks J, Unadkat J, Raimondi G, Wang Z, Ikeguchi R, et al. Rapamycin-conditioned, alloantigen-pulsed dendritic cells promote indefinite survival of vascularized skin allografts in association with $T$ regulatory cell expansion. Transpl Immunol. 2008;18:307-18.

38. Raimondi G, Sumpter TL, Matta BM, Pillai M, Corbitt N, Vodovotz Y, et al. Mammalian target of rapamycin inhibition and alloantigen-specific regulatory $T$ cells synergize to promote long-term graft survival in immunocompetent recipients. J Immunol. 2010;184:624-36.

39. Bonham CA, Peng L, Liang $X$, Chen Z, Wang L, Ma L, et al. Marked prolongation of cardiac allograft survival by dendritic cells genetically engineered with NF-kappa B oligodeoxyribonucleotide decoys and adenovira vectors encoding CTLA4-Ig. J Immunol. 2002;169:3382-91.

40. Chorny A, Gonzalez-Rey E, Fernandez-Martin A, Ganea D, Delgado M. Vasoactive intestinal peptide induces regulatory dendritic cells that prevent acute graft-versus-host disease while maintaining the graft-versus-tumor response. Blood. 2006;107:3787-94.

41. Thomson AW, Robbins PD. Tolerogenic dendritic cells for autoimmune disease and transplantation. Ann Rheum Dis. 2008;67(3):iii90-6.

42. Zhou $\sqcup$, Tedder TF. CD14+ blood monocytes can differentiate into functionally mature CD83+ dendritic cells. Proc Natl Acad Sci U S A. 1996;93:2588-92.

43. Banchereau J, Ueno H, Fay JW, Palucka AK. Dendritic Cells as Therapeutic Vaccines in Cancer. J Immunother. 2009;32:980.

44. Schuler-Thurner B, Dieckmann D, Keikavoussi P, Bender A, Maczek C, Jonuleit $\mathrm{H}$, et al. Mage-3 and influenza-matrix peptide-specific cytotoxic $\mathrm{T}$ cells are inducible in terminal stage HLA-A2.1+ melanoma patients by mature monocyte-derived dendritic cells. J Immunol. 2000;165:3492-6.

45. Hart DN, Hill GR. Dendritic cell immunotherapy for cancer: application to low-grade lymphoma and multiple myeloma. Immunol Cell Biol. 1999;77:451-9.

46. Wierecky J, Müller MR, Wirths S, Halder-Oehler E, Dorfel D, Schmidt SM, et al. Immunologic and clinical responses after vaccinations with peptide-pulsed dendritic cells in metastatic renal cancer patients. Cancer Res. 2006;66:5910-8.

47. Gao D, Li C, Xie X, Zhao P, Wei X, Sun W, et al. Autologous tumor lysate-pulsed dendritic cell immunotherapy with cytokine-induced killer cells improves survival in gastric and colorectal cancer patients. Plos One. 2014;9:e93886.

48. Vik-Mo EO, Nyakas M, Mikkelsen BV, Moe MC, Due-Tonnesen P, Suso EM, et al. Therapeutic vaccination against autologous cancer stem cells with mRNA-transfected dendritic cells in patients with glioblastoma. Cancer Immunol Immunother. 2013;62:1499-509.

49. Mahon FX, Rea D, Guilhot J, Guilhot F, Huguet F, Nicolini F, et al. Discontinuation of imatinib in patients with chronic myeloid leukaemia who have maintained complete molecular remission for at least 2 years: the prospective, multicentre Stop Imatinib (STIM) trial. Lancet Oncol. 2010;11:1029-35.

50. Kawai T, Akira S. Toll-like receptors and their crosstalk with other innate receptors in infection and immunity. Immunity. 2011;34:637-50.

51. Ozes ON, Mayo LD, Gustin JA, Pfeffer SR, Pfeffer LM, Donner DB. NF-kappaB activation by tumour necrosis factor requires the Akt serine-threonine kinase. Nature. 1999;401:82-5.
52. Demarchi F, Bertoli C, Sandy P, Schneider C. Glycogen synthase kinase-3 beta regulates NF-kappa B1/p105 stability. J Biol Chem. 2003;278:39583-90

53. Brossart P, Zobywalski A, Grünebach F, Behnke L, Stuhler G, Reichardt VL, et al. Tumor necrosis factor alpha and CD40 ligand antagonize the inhibitory effects of interleukin 10 on T-cell stimulatory capacity of dendritic cells. Cancer Res. 2000;60:4485-92.

54. Lundberg K, Albrekt AS, Nelissen I, Santegoets S, de Gruijl TD, Gibbs S, et al. Transcriptional profiling of human dendritic cell populations and models-unique profiles of in vitro dendritic cells and implications on functionality and applicability. Plos One. 2013;8:e52875.

55. Cwynarski K, Laylor R, Macchiarulo E, Goldman J, Lombardi G, Melo JV, et al. Imatinib inhibits the activation and proliferation of normal T lymphocytes in vitro. Leukemia. 2004;18:1332-9.

56. Dietz AB, Souan L, Knutson GJ, Bulur PA, Litzow MR, Vuk-Pavlovic S. Imatinib mesylate inhibits T-cell proliferation in vitro and delayed-type hypersensitivity in vivo. Blood. 2004;104:1094-9.

57. Seggewiss R, Lore K, Greiner E, Magnusson MK, Price DA, Douek DC, et al. Imatinib inhibits T-cell receptor-mediated T-cell proliferation and activation in a dose-dependent manner. Blood. 2005;105:2473-9.

58. Zhou LT, Liu FY, Li Y, Peng YM, Liu YH, Li J. Gpnmb/osteoactivin, an attractive target in cancer immunotherapy. Neoplasma. 2012;59:1-5.

59. Hwu P, Sznol M, Pavlick A, Kluger H, Kim KB, Boasberg P, et al. A phase I/II study of CR011-vCMMAE, an antibody-drug conjugate (ADC) targeting glycoprotein NMB (GPNMB) in patients (pts) with advanced melanoma. ASCO Meeting Abstracts. 2009;27:9032.

60. Hamid O, Sznol M, Pavlick AC, Kluger HM, Kim KB, Boasberg PD, et al. Frequent dosing and GPNMB expression with CDX-011 (CR011-vcMMAE), an antibody-drug conjugate (ADC), in patients with advanced melanoma. ASCO Meeting Abstracts. 2010;28:8525.

61. Yardley DA, Weaver R, Melisko ME, Saleh MN, Arena FP, Forero A, et al. A randomized phase 2 study of the antibody-drug conjugate CDX-011 in advanced GPNMB-overexpressing breast cancer: The EMERGE study. Cancer Res. 2012;72:6-10.

62. Qian X, Mills E, Torgov M, LaRochelle WJ, Jeffers M. Pharmacologically enhanced expression of GPNMB increases the sensitivity of melanoma cells to the CR011-vCMMAE antibody-drug conjugate. Mol Oncol. 2008;2:81-93.

\section{Submit your next manuscript to BioMed Central and take full advantage of:}

- Convenient online submission

- Thorough peer review

- No space constraints or color figure charges

- Immediate publication on acceptance

- Inclusion in PubMed, CAS, Scopus and Google Scholar

- Research which is freely available for redistribution 